\title{
Integración del diseño e implementación de la electrónica de una plataforma robótica educativa multidisciplinar como soporte al aprendizaje
}

\begin{abstract}
Daniel Aponte Núñez ${ }^{\mathrm{a}}$, Eduardo Quevedo Gutiérrez ${ }^{\mathrm{b}}$, Pedro Hernández Castellano ${ }^{\mathrm{c}}$, Alberto Zapatera Llinares ${ }^{\mathrm{d}}$, Himar Fabelo Gómez ${ }^{\mathrm{e}}$, Samuel Ortega Sarmiento ${ }^{\mathrm{f}}$ y Gustavo Marrero Callicó ${ }^{\mathrm{g}}$

${ }^{a}$ Escuela de Ingenierías Industriales y Civiles, Universidad de Las Palmas de Gran Canaria, daniel.aponte101@alu.ulpgc.es ${ }^{\mathrm{b}}$ Instituto Universitario de Microelectrónica Aplicada, Universidad de Las Palmas de Gran Canaria, equevedo@iuma.ulpgc.es ${ }^{\mathrm{C}}$ Grupo de Investigación de Fabricación Integrada y Avanzada, Universidad de Las Palmas de Gran Canaria, pedro.hernandez@ulpgc.es ${ }^{\mathrm{d}}$ Departamento de Educación, Universidad CEU Cardenal Herrera, alberto.zapatera@uchceu.es ${ }^{\text {In }}$, ustituto Universitario de Microelectrónica Aplicada, Universidad de Las Palmas de Gran Canaria, hfabelo@iuma.ulpgc.es ${ }^{\mathrm{f}}$ Instituto Universitario de Microelectrónica Aplicada, Universidad de Las Palmas de Gran Canaria, sortega@iuma.ulpgc.es ${ }^{\mathrm{g}}$ Instituto Universitario de Microelectrónica Aplicada, Universidad de Las Palmas de Gran Canaria, gustavo@iuma.ulpgc.es
\end{abstract}

\begin{abstract}
The implementation of robotics in schools is inevitable in the years to come. Currently, this integration is not feasible for all schools (especially public ones) due to the high economic cost, which in most cases offer a closed system (both hardware and software) which limits the robot to a single educational level.
\end{abstract}

The educational innovation project "ROBOT-EDULPGC, Design, implementation and implementation of a low-cost modular educational robotics platform" of the University of Las Palmas de Gran Canaria, seeks to offer an educational platform designed for use at all educational levels (multidisciplinary), with free hardware and software, and low cost, thus eliminating the economic barrier.

This work reflects the results of a statistical study carried out on students of different engineering degrees, in particular those of the Degree in Industrial and Automatic Electronic Engineering, all belonging to the School of Industrial and Civil Engineering at the University of Las Palmas de Gran Canaria. A specific survey has been carried out, designed specifically to evaluate the integration of robotics in their degree as a teaching tool and on project-based teaching as opposed to traditional teaching.

Keywords: innovation, education, robot, robotics, modular, hardware, software, engineering, electronic, university

\section{Resumen}

La implementación de la robótica en los centros de enseñanza es algo inevitable en los años venideros. Actualmente, esta integración no es asumible por todos los centros (especialmente los públicos) debido al elevado coste económico, que en la mayoría de las ocasiones ofrecen un sistema cerrado (tanto en hardware como software) lo que limita el robot a un solo nivel educativo.

El proyecto de innovación educativa "ROBOT-EDULPGC, Diseño, implementación y puesta en práctica de una plataforma modular de robótica educativa de bajo coste" de la Universidad de Las Palmas de Gran Canaria, busca ofrecer una plataforma educativa pensada para usarse en 
todos los niveles educativos (multidisciplinar), con hardware y software libre, y de bajo coste eliminando así la barrera económica.

Este trabajo refleja los resultados de un estudio estadístico realizado a estudiantes de diferentes titulaciones de ingeniería, en particular a los del Grado en Ingeniería Electrónica Industrial y Automática, todos pertenecientes a la Escuela de Ingenieros Industriales y Civiles en la Universidad de Las Palmas de Gran Canaria. Se ha realizado una encuesta diseñada especificamente para evaluar la integración de la robótica en su titulación como herramienta didáctica y sobre la enseñanza basada en proyectos frente a la tradicional.

Palabras clave: innovación, educación, robot, robótica, modular, hardware, software, ingeniería, electrónica, universidad

\section{Introducción}

En la última década, la robótica modular en los centros de enseñanza, ha sufrido un cambio drástico en lo que a implementación e integración se refiere, de ser prácticamente inexistente se ha pasado a que, en la actualidad, cada vez más centros educativos implementan la robótica como herramienta en sus planes docentes (Pinto et al, 2009)

Para hablar de robótica y su modularidad es imprescindible comprender que los microcontroladores y su programación son un "pack" indivisible, esto se debe, a que el hardware (el microcontrolador) sin la programación (el software) es solo una agrupación de componentes electrónicos estáticos, es decir, sin función alguna. (Vega-Moreno et al, 2015)

En el caso de los dispositivos electrónicos en los centros educativos sucede algo similar a la robótica, pero menos drástico. En la actualidad, los estudiantes y profesores disponen en la actualidad de ordenadores portátiles y tabletas como herramientas de apoyo a la docencia. Este escenario dista mucho del de hace unos años, donde solo se disponía de ordenadores fijo usados en las horas destinadas a laboratorio (Alcaide y De la Poza, 2019).

En docencia se diferencian dos tipos de uso de la programación y la robótica como apoyo en el aula: por un lado, la robótica y la programación educativa consisten en un conjunto de elementos físicos o de programación que motivan al alumnado a construir, programar, razonar de manera lógica y crear nuevas interfaces o dispositivos; por otro, la programación y la robótica como elemento social, por ejemplo a modo de juego, de forma que sistemas autónomos o semiautónomos interactúan con humanos u otros agentes físicos o software en roles como entrenador, compañero, dispositivo tangible o registro de información.” (Angulo, 2017)

La robótica y programación usada para crear interfaces o nuevos dispositivos, es la más beneficiosa en el ámbito de las STEAM (ciencia, ingeniería, arte y matemáticas) puesto que en carreras de este ámbito es habitual que el plan docente enmarque la robótica y la programación, aunque en niveles mucho más contenidos de los que propone este estudio (Quevedo et al, 2018)

El proyecto de innovación educativa "ROBOT-EDULPGC, Diseño, implementación y puesta en práctica de una plataforma modular de robótica educativa de bajo coste" aprobado por la Universidad de Las Palmas de Gran Canaria (ULPGC). Este proyecto tiene como finalidad el diseño e implantación de una plataforma robótica que abarque todos los niveles educativos (desde infantil hasta la universidad) para ser usada como apoyo a la docencia. Donde la modularidad, la multidisciplinariedad y el bajo coste son las 
tres "patas" que fundamentan este proyecto de innovación que busca colarse en todos los centros de enseñanza en los próximos años.

A lo largo del presente artículo se expondrán los resultados de un estudio realizado a alumnos de la Escuela de Ingenieros Industriales y Civiles (EIIC) de la ULPGC donde se engloba a la mayor parte de las titulaciones impartidas en la EIIC.

El estudio refleja el interés y motivación que les despierta a los estudiantes la implantación de un sistema de enseñanza distinto al tradicional, basado no sólo en el uso de la robótica como herramienta de apoyo, sino basada en proyectos mediante la integración de las diferentes asignaturas de la titulación.

Cabe destacar que la integración de la electrónica y robótica en las aulas es una realidad gracias al gran interés y compromiso de los docentes que se atreven a embarcarse en esta aventura de la robótica educativa. Es por ello, que sin ser del todo obligada salvo en algunas asignaturas específicas, el profesorado realiza esta implementación por iniciativa propia y no por imposición del centro o escuela donde se realiza la docencia.

\section{Objetivos}

Este documento tiene como objetivo principal el análisis de los resultados obtenidos de un estudio estadístico realizado a 78 estudiantes de diferentes Grados de Ingenierías, donde 33 de ellos pertenecen al Grado de Ingeniería Electrónica Industrial y Automática (grupo de estudio) y los 45 restantes reparten sus matrículas en las otras titulaciones de la escuela (grupo de control). El estudio hace referencia a integración e implantación de la robótica en su plan académico, con el fin de establecer si es viable la implantación de dicha tecnología en la docencia.

Por otra parte, otro de los objetivos del estudio, es evaluar si despertaría un mayor interés en los estudiantes una enseñanza basada en proyectos donde las asignaturas de la titulación mediante la coordinación e integración de diferentes asignaturas de la titulación con el fin de proponer una modificación en el tipo de docencia de la Escuela de Ingenieros Industriales y Civiles (EIIC).

\section{Desarrollo de la innovación}

Para el desarrollo del estudio y el diseño de la innovación de este trabajo, realizado con los alumnos del Grado en Ingeniería Electrónica Industrial y Automática, se ha elaborado un marco contextual, así como dos puntos de vista bien diferenciados y cruciales para su fundamentación. El primero, el punto de vista didáctico, se basa en el impacto que tendría en la docencia la integración e implantación de la robótica en las diferentes asignaturas de la titulación. El segundo (el técnico), ha estudiado la visión de los estudiantes respecto a la implementación de dicha tecnología.

Haciendo uso de un cuestionario con preguntas específicas y que diferencia a los alumnos del Grado de Electrónica y Automática (grupo de estudio, 33) frente a los de otras ingenierías (grupo de control, 45) se han recabado y analizado los datos con los que se expondrán los siguientes apartados de este trabajo.

\subsection{Contexto}

En la actualidad, existe gran variedad de robots con fines educativos, aunque todos comparten ámbito de aplicación (el educativo), son muy diferentes entre sí, pero no por ello dejan de compartir características. A continuación, y con el fin de "filtrar" los robots, se agruparán aquellos que posean las mismas 
características técnicas y funcionalidades en grupos enumerados del 1 al 3. EL grupo 1 corresponde a los robots que tienen tanto hardware como software cerrado, el grupo 2, sólo hardware o software cerrado, y los del grupo 3, son completamente abiertos (la opción que nos interesa para el desarrollo del "ROBOTEDULPGC" que se menciona en el apartado técnico). A continuación, en la Tabla 1, se representan estos grupos de manera más clara.

Tabla 1: Tabla de hardware y software cerrado

\begin{tabular}{|c|c|c|c|}
\hline Grupo & Robot & $\begin{array}{c}\text { Hardware } \\
\text { cerrado }\end{array}$ & $\begin{array}{c}\text { Software } \\
\text { cerrado }\end{array}$ \\
\hline \multirow{7}{*}{1} & COJI & $\mathbf{X}$ & $\mathbf{X}$ \\
\hline & CODER MIP & $\mathbf{X}$ & $\mathbf{X}$ \\
\hline & NEXT 2.0 & $\mathbf{X}$ & $\mathbf{X}$ \\
\hline & RATÓN ROBOT & $\mathbf{X}$ & $\mathbf{X}$ \\
\hline & DOC & $\mathbf{X}$ & $\mathbf{X}$ \\
\hline & BEE-BOT & $\mathbf{X}$ & $\mathbf{X}$ \\
\hline & PRO-BOT & $\mathbf{X}$ & $\mathbf{X}$ \\
\hline \multirow{3}{*}{2} & LEGO-BOOST & & $\mathbf{X}$ \\
\hline & MINDSTORMS & & $\mathbf{X}$ \\
\hline & AISOY 1 & $\mathbf{X}$ & \\
\hline \multirow{7}{*}{3} & ESCORNABOT & & \\
\hline & MAQUEEN + BBC & & \\
\hline & micro: bit & & \\
\hline & THYMIO & & \\
\hline & ZOWI & & \\
\hline & mBOT & & \\
\hline & EDISON & & \\
\hline
\end{tabular}

En la Tabla 1 se hace una comparativa de 16 robots, 7 de ellos pertenecen al grupo 1: COJI (Wowwee, 2016), CODER MIP (Juguetrónica, 2016), NEXT 2.0 (Edelvides, 2019), RATÓN ROBOT (robot para niños, 2017), DOC (Clementoni, 2017), BEE-BOT y PRO-BOT (Ro-botica, 2015). En el grupo 2 (hardware o software cerrado) se encuentra a LEGO-BOOST, MINDSTORMS (LEGO, 2014 y 2018) y a AISOY 1 (Aisoy España, 2009). En la parte baja de la Tabla 1 se encuentra a ESCORNABOT, MAQUEEN + BBC micro: bit (DfRobots, 2018), THYMIO, ZOWI (bq, 2015), mBOT (Makeblock, 2012) y el robot Edison (Microbric, 2014)

En el apartado hardware, lo más relevante o destacable para que la robótica pueda ser implementada en las aulas como herramienta de apoyo, es que el robot posea modularidad, es decir, que se pueda ampliar o reducir el número de elementos mecánicos y electrónicos como sensores o actuadores, en función de las necesidades del nivel académico en que se integra. Ello consigue, que el robot sea flexible y, por tanto, válido para distintos niveles educativos (multidisciplinariedad).

El apartado software es algo más complejo, existen diferentes aspectos a tener en cuenta como:

- El lenguaje de programación.

- Si la programación es por bloques o no.

- El entorno de programación.

- Las plataformas para las que está disponible el software.

(c)) EY-NC-ND 2020, Universitat Politècnica de València Congreso In-Red (2020) 
La elección del lenguaje de programación usado para controlar el comportamiento del robot es un factor determinante para el nivel educativo al que está dirigido. Para los primeros niveles de infantil y primaria se usa algo que se denomina "programación por bloques", que consiste en usar bloques que "contienen" una programación preestablecida, sin necesidad de saber programar de manera directa. Esto es realmente efectivo para los estudiantes que se están iniciando en la programación y tiene la versatilidad de extrapolarse a mayores, puesto que ayuda a estructurar y realizar un código de programación. El entorno de programación es el software usado para realizar la programación del robot, ya sea mediante bloques (Scratch, Bitbloq, S4A, LabVIEW) o en alguno de los muchos lenguajes existentes (C, C++, C\#, Java). A la hora de diseñar un robot modular programable educativo, es muy importante que se piense que llegar a la mayoría de centros de enseñanza, y que no necesiten realizar un desembolso extra, más que el del robot, por eso lo más responsable sería que la interfaz que gobierna el robot, este orientada a un entorno Windows, puesto que todos los centros educativos disponen de ordenadores en sus instalaciones.

A continuación, en la Tabla 2, se recoge en una comparativa los robots que conforman este estado del arte. En ella se presentan características técnicas como la edad recomendada, el precio o la modularidad, esencial en una plataforma educativa, puesto que sin ella, desaparece la multidisciplinariedad. También se incluye el tipo de alimentación del dispositivo (batería recargable o pilas), esto a priori puede parecer una característica banal, pero en la integración y sobre todo en el largo plazo cobra gran relevancia. El uso de baterías tiene ventajas respecto al uso de pilas desechables convencionales (ahorro económico) e inconvenientes, pues se van deteriorando, con las cargas y descargas habituales del uso, no hay que olvidar que el uso de baterías obliga a los docentes a prever que esté cargada a la hora de realizar la docencia. Las pilas son la mejor opción para la robótica educativa, puesto que cambiándolas cuando se agoten podemos tener el robot funcionando el tiempo que sea necesario, sin tiempo de espera por carga, además de reducir el coste de manera significativa.

La Tabla 2 también destaca el software usado por los dispositivos, así como si dispone de programación por bloques ya sea haciendo uso de software libre o con aplicación propia cerrada. Todos los robots que se incluyen en la tabla admiten la programación por bloques, independiente del software que usen, el más versátil es el robot "ESCORNABOT" puesto que se basa en una placa Arduino (ESCORNABOT, 2015) que admite una gran variedad de software y lenguajes para su programación (Arduino,2006). Los robots recogidos en la Tabla 2 que no permiten la programación por bloques, tampoco permiten la programación mediante software, es decir, que solo se pueden programar sus movimientos en el propio dispositivo haciendo uso de los botones de dirección, este tipo de robot suele usarse en el primer nivel educativo, donde los niños hacen uso de tapetes temáticos y el robot se desplaza por encima de ellos como método de aprendizaje.

\subsection{Aportación didáctica}

Al margen del debate existente entre los docentes sobre el material que debe utilizarse en el aula, merece ser analizada la opinión de los alumnos. Algunos investigadores afirman que los dispositivos tangibles (robots en nuestro caso) aumentan el nivel de inmersión debido a que observan una aplicación inmediata de la actividad que están realizando. Por otra parte, la robótica educativa fomenta el desarrollo de la creatividad, además de algunas habilidades de pensamiento, las cuales se articulan con: la solución de problemas, el manejo de herramientas, el manejo de circuitos y la programación entre otros (Peralta, 2015) 
Integración del diseño e implementación de la electrónica de una plataforma robótica educativa multidisciplinar como soporte al aprendizaje

\begin{tabular}{|c|c|c|c|c|c|c|c|c|}
\hline Fabricante & Modelo & $\begin{array}{l}\text { Aliment: } \\
\text { Baterías }\end{array}$ & $\begin{array}{l}\text { ción } \\
\text { Pilas }\end{array}$ & Modularidad & Software & $\begin{array}{c}\text { Programación } \\
\text { por bloques }\end{array}$ & Edad & $\begin{array}{c}\text { Precio } \\
(€)\end{array}$ \\
\hline Aisoy & AISOY 1 & $\mathrm{X}$ & & & S4A & $\mathrm{X}$ & +3 & 279 \\
\hline WowWee & COJI & & $\mathrm{X}$ & & SP & $\mathrm{X}$ & +4 & 59,90 \\
\hline JUGUETRÓNICA & CODER MIP & $\mathrm{X}$ & & & SP & $\mathrm{X}$ & +6 & 99 \\
\hline BQ & ZOWI & $\mathrm{X}$ & & & Bitbloq & $\mathrm{X}$ & +8 & 79,90 \\
\hline EDELVIVES & NEXT 2.0 & $\mathrm{X}$ & & & $\mathrm{SP}+\mathrm{D}$ & $\mathrm{X}$ & +3 & 131,04 \\
\hline $\begin{array}{l}\text { Learning } \\
\text { Resources }\end{array}$ & $\begin{array}{l}\text { RATÓN } \\
\text { ROBOT }\end{array}$ & & $\mathrm{X}$ & & D & & +4 & 32,95 \\
\hline Clementoni & DOC & & $\mathrm{X}$ & & D & & +5 & 29,99 \\
\hline Makeblock & mBOT & & $\mathrm{X}$ & $\mathrm{X}$ & $\begin{array}{c}\text { Mbot } \\
+ \text { Bitbloq }\end{array}$ & $\mathrm{X}$ & +10 & 89,99 \\
\hline Edison & EDISON & & $\mathrm{X}$ & $\mathrm{X}$ & SP & $\mathrm{X}$ & +4 & 55 \\
\hline $\begin{array}{l}\text { DFROBOT } \\
\text { micro: bit }\end{array}$ & $\begin{array}{l}\text { MAQUEEN + } \\
\text { BBC micro: bit }\end{array}$ & & $\mathrm{X}$ & $\mathrm{X}$ & $\begin{array}{l}\text { JAVA+ } \\
\text { PYTHON }\end{array}$ & $\mathrm{X}$ & +4 & 47,34 \\
\hline LEGO & LEGO-BOOST & & $\mathrm{X}$ & $\mathrm{X}$ & SP & $\mathrm{X}$ & +7 & 159,90 \\
\hline LEGO & MINDSTORMS & $\mathrm{X}$ & & $\mathrm{X}$ & SP & $\mathrm{X}$ & +10 & 491 \\
\hline ESCORNABOT & ESCORNABOT & & $\mathrm{X}$ & $\mathrm{X}$ & $\begin{array}{c}\text { S4A } \\
\text { Bitbloq } \\
\text { Arduino }\end{array}$ & $\mathrm{X}$ & +4 & 26,90 \\
\hline THYMIO & THYMIO & $\mathrm{X}$ & & $\mathrm{X}$ & $\begin{array}{c}\text { Scratch }+ \\
\text { SP }\end{array}$ & $\mathrm{X}$ & +6 & 120 \\
\hline $\begin{array}{l}\text { RO-BOTICA } \\
\text { Global }\end{array}$ & BEE-BOT & $\mathrm{X}$ & & & D & & +3 & 85 \\
\hline $\begin{array}{c}\text { RO-BOTICA } \\
\text { Global }\end{array}$ & PRO-BOT & $\mathrm{X}$ & $\mathrm{X}$ & & D & & +8 & 169 \\
\hline
\end{tabular}

No es comparable que los alumnos realicen un aprendizaje basado únicamente en un conocimiento con aplicaciones abstractas, a que experimenten con ese conocimiento haciendo uso de objetos tangibles desarrollando ese trabajo abstracto, por ejemplo, no es comparable que los estudiantes elaboren un código capaz de controlar la velocidad de movimiento del eje motriz de un robot y no lo vean de manera física, a que se materialice y que tras finalizar el programa puedan observar en el laboratorio como el código que ellos han creado, efectivamente no sólo funciona, sino que es de utilidad en el mundo real. 


\subsection{Aportación técnica}

La plataforma robótica planteada en el proyecto de innovación educativa de referencia plantea hardware y software abierto. Esto se consigue mediante el modularidad y usando microcontroladores que permiten usar diferentes soluciones softwares para su programación, de lo contrario, no cumpliría la condición de multidisciplinariedad y no serviría para todos los niveles educativos como se pretende.

El aspecto económico es limitante para que muchos centros de enseñanza implementen la robótica en sus planes docentes, por eso, esta plataforma persigue el objetivo de ofrecer versatilidad, modularidad y software libre a un precio contenido. Como se pudo ver en la Tabla 2, había robots que la relación preciofuncionalidad era bastante mala, puesto que su precio era muy elevado para las características que ofrecía. La mayoría de estor robots no disponían modularidad y su software no era libre, y su precio rondaban los cientos de euros.

Lo que se espera de esta plataforma al término de su diseño, es que se obtenga un robot, con un aspecto físico parecido al del robot "Thymio" (ver Figura 1), donde la plataforma contaría con un "cuerpo" que contendría elementos básicos para el funcionamiento del robot, como puede ser el microcontrolador, las baterías o los ejes motrices, y unos conectores universales donde se pe pudieran añadir sensores y actuadores según lo requiera la docencia (MOBSYA, 2011).

El proyecto de innovación educativa planteado busca englobar las mejores virtudes de los robots actuales en un único dispositivo a un precio muy contenido, con el fin de que todos los centros educativos de todos los niveles cuenten con ella para apoyar la labor docente.

Por otra parte, si se habla de integrar un nuevo método de enseñanza (por proyectos con integración de diferentes asignaturas) y con un material distinto al tradicional (la robótica modular) se debe tener en cuenta que esto supone un sobreesfuerzo tanto para docentes, como para estudiantes puesto que ninguno está acostumbrado a este método de enseñanza, lo que supone un obstáculo a salvar para su implantación.

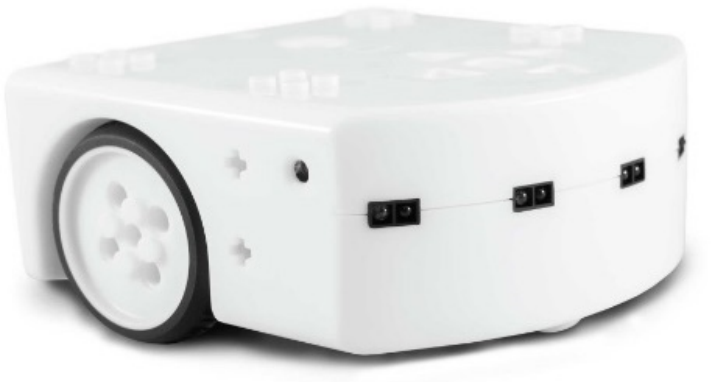

Fig. 1 Robot Thymio

\section{Resultados}

Una vez presentados el diseño de la innovación planteada desde un punto de vista didáctico y técnico se presentan las expectativas iniciales de un conjunto de estudiantes del Grado en Electrónica Industrial y Automática de la ULPGC, a través de una encuesta específicamente diseñado para ello. En la titulación comentada (grupo de estudio) se pretende integrar la electrónica y la programación de la plataforma robótica educativa modular descrita en la docencia, si bien se plantea también la encuesta a alumnos de otras titulaciones (grupo de control), obteniendo una muestra total de 78 estudiantes, distribuidos tal y como se presenta en la Tabla 3. Dentro del apartado de "Resto de titulaciones" se incluyen los grados de Ingeniería Geomática, Ingeniería Civil, Ingeniería Naval e Ingeniería en Organización Industrial. 
Tabla 3. Muestra de estudiantes que respondieron a la encuesta planteada

\begin{tabular}{cc}
\hline Titulación & N (Tamaño de la muestra) \\
\hline $\begin{array}{c}\text { Grado en Electrónica Industrial y Automática } \\
\text { (Grupo de estudio) } \\
\text { Otras titulaciones } \\
\text { (Grupo de control) }\end{array}$ & $\mathbf{3 3}$ \\
Grado en Diseño Industrial y Desarrollo de Productos & $\mathbf{4 5}$ \\
Grado en Ingeniería Química & 20 \\
Grado en Ingeniería Química Industrial & 11 \\
Grado en Ingeniería Eléctrica & 5 \\
Resto de titulaciones & 5 \\
\hline
\end{tabular}

Como preguntas iniciales de la encuesta para establecer un contexto general del punto de partida se requirió la edad de los participantes, el curso más alto en el que estuviera matriculado el estudiante y los conocimientos de robótica. Con respecto a la edad resultaron valores medios de 21,42 para el grupo de estudio y de 21,64 para el grupo de control, por lo que no existe una diferencia estadísticamente significativa ( $\mathrm{p}=0.6812$ según test de $\mathrm{T}$ de Student, para el resto de variables se ha realizado test de Chicuadrado al tratarse de variables discretas). Por otra parte, si bien no existe diferencia estadísticamente significativa en lo que se refiere al curso más alto de matrícula del estudiante ( $\mathrm{p}=0.5906)$, estando la mayoría de los alumnos matriculados en $4^{\circ}$ curso, como se presenta en la Figura 2, existe una variabilidad clara en los conocimientos de robótica que los alumnos declaran $(\mathrm{p}=0.0145)$, para los cuales se han establecido 4 categorías, tal y como se presenta en la Figura 2:

- Nulos.

- Básicos (juguetes educativos).

- Medios (programación por bloques).

- Avanzados (conocimientos sobre construcción, conocimientos sobre más de un lenguaje de programación).

Se observa que más del 50\% de los alumnos del Grado de Electrónica y Automática Industrial declaran disponer de conocimientos avanzados o medios de robótica, mientras que cerca del 50\% de los alumnos de otras titulaciones expresan que sus conocimientos son nulos.
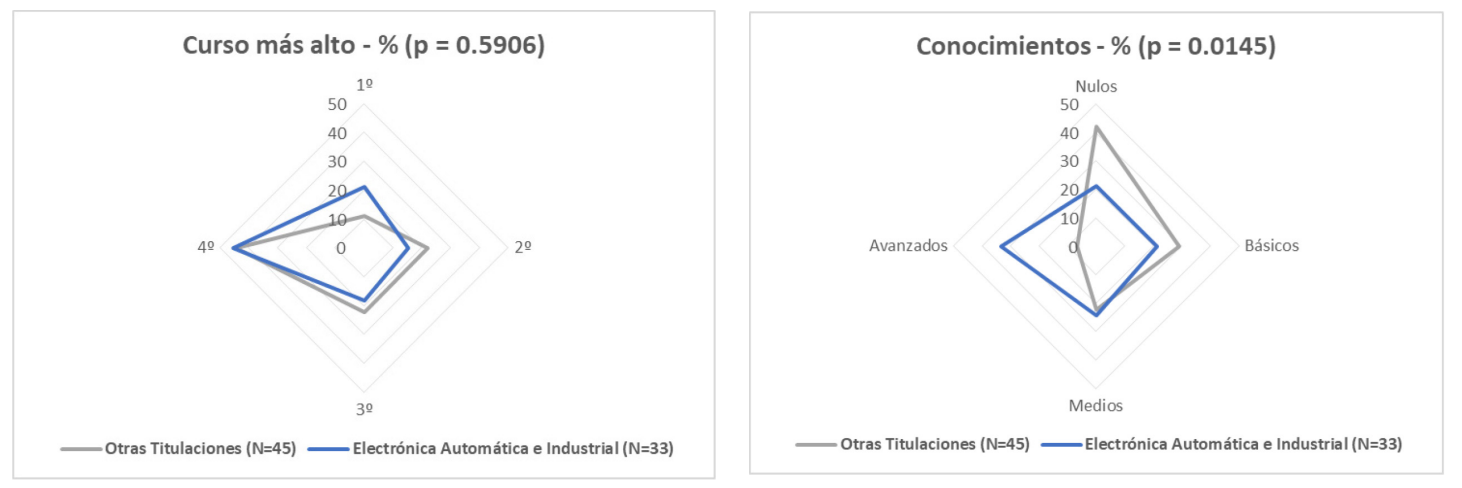

Fig. 2 Resultados porcentuales de preguntas de contexto general: curso más alto (izquierda) y conocimientos de robótica (derecha) 
El resto de cuestiones de la encuesta conforman un total de 16 preguntas. 12 de estas preguntas evaluadas según la escala Likert, que se presentan en la Tabla 4, asignando un identificador a cada pregunta. En la Tabla 5 se presentan los promedios del grupo de estudio (Grado de Ingeniería Electrónica y Automática) y el grupo de control (otras titulaciones), así como el p valor que determina si existen diferencias estadísticamente significativas entre grupos, concluyendo que existen si $\mathrm{p}<0.05$ para un nivel de confianza del 95\%. Las restantes 4 preguntas (abiertas) son relativas a las asignaturas de aplicación, ventajas y desventajas del uso de la robótica y a cualquier cuestión a añadir.

Tabla 4. 12 preguntas evaluadas en escala Likert, asignando un identificador a cada una de ellas

\begin{tabular}{|c|c|}
\hline Identificador & Pregunta \\
\hline Formación & Me gustaría recibir conocimientos sobre robótica \\
\hline Importancia & $\begin{array}{l}\text { Creo que los conocimientos sobre robótica son } \\
\text { imprescindibles en el currículo de cualquier Ingeniero }\end{array}$ \\
\hline Aplicación & $\begin{array}{l}\text { Creo que es posible diseñar un robot que de forma modular } \\
\text { pueda aplicarse desde niveles de Infantil hasta universitarios }\end{array}$ \\
\hline Instrucciones & $\begin{array}{l}\text { Considerando un robot a construir, prefiero disponer de unas } \\
\text { instrucciones pautadas a construirlo a partir de mis propias ideas }\end{array}$ \\
\hline Inconvenientes & $\begin{array}{l}\text { Los docentes tendrían inconvenientes para integrar } \\
\text { la robótica modular en las asignaturas que imparten }\end{array}$ \\
\hline Electrónica & $\begin{array}{l}\text { Sería sencillo integrar el diseño de la electrónica de } \\
\text { un robot en las asignaturas de mi titulación. }\end{array}$ \\
\hline Programación & $\begin{array}{l}\text { Sería sencillo integrar la programación de } \\
\text { un robot en las asignaturas de mi titulación. }\end{array}$ \\
\hline C. Mecánicos & $\begin{array}{l}\text { Sería sencillo integrar el diseño de componentes mecánicos } \\
\text { para un robot en las asignaturas de mi titulación. }\end{array}$ \\
\hline C. Estructurales & $\begin{array}{l}\text { Sería sencillo integrar la búsqueda y el análisis de componentes } \\
\text { estructurales para un robot en las asignaturas de mi titulación. }\end{array}$ \\
\hline Coordinación & $\begin{array}{l}\text { Un proyecto de robótica conjunto es un buen medio para fomentar } \\
\text { la integración y la coordinación entre distintas asignaturas }\end{array}$ \\
\hline $\mathrm{ABP}$ & $\begin{array}{l}\text { Prefiero una orientación de aprendizaje basado en } \\
\text { proyectos frente a otra aproximación más tradicional }\end{array}$ \\
\hline Integración & $\begin{array}{l}\text { Las asignaturas de mi titulación podrían incluir la construcción de un robot } \\
\text { como proyecto sin tener que distanciarse del temario a impartir }\end{array}$ \\
\hline
\end{tabular}

Tabla 5. Evaluaciones promedio de grupos de estudio y control y p-valor del test de Chi-Cuadrado

\begin{tabular}{ccccc}
\hline Identificador & $\begin{array}{c}\text { Grupo de Estudio } \\
\text { (Promedio, N=33) }\end{array}$ & $\begin{array}{c}\text { Grupo de Control } \\
\text { (Promedio, N=45) }\end{array}$ & $\begin{array}{c}\text { Diferencia de Promedios } \\
\text { G. Estudio - G. Control }\end{array}$ & p \\
\hline Formación & 4,76 & 3,64 & 1,12 & $<0.0001$ \\
Importancia & 4,03 & 3,36 & 0,67 & 0.0237 \\
Aplicación & 4,61 & 3,62 & 0,98 & 0.0011 \\
Instrucciones & 3,48 & 3,44 & 0,04 & 0.5121 \\
Inconvenientes & 3,03 & 3,73 & $-0,70$ & 0.0386 \\
Electrónica & 4,42 & 2,80 & 1,62 & $<0.0001$ \\
Programación & 4,42 & 2,80 & 1,62 & $<0.0001$ \\
C. Mecánicos & 4,27 & 3,33 & 0,94 & 0.0042 \\
C. Estructurales & 4,15 & 3,04 & 1,11 & $<0.0001$ \\
Coordinación & 4,36 & 3,49 & 0,87 & 0.0005 \\
ABP & 4,36 & 3,86 & 0,50 & 0.1495 \\
Integración & 4,24 & 2,98 & 1,26 & 0.0001 \\
\hline
\end{tabular}


Los resultados a las 12 preguntas de escala Likert, presentados en la Tabla 4 y analizados en más detalle en la Figura 3 haciendo uso de diagramas radiales, demuestran que los conocimientos de robótica con los que ya parten los ingenieros del grupo de estudio les hacen ser más proclives al planteamiento de un proyecto de robótica educativa en su titulación frente al resto de titulaciones. Este hecho es especialmente relevante en factores como el deseo de recibir aún más conocimientos de robótica (Formación) y a no ver complicado la integración de la electrónica y la programación (diferencia de promedios de 1.62 puntos en estos dos factores), así como los componentes estructurales en un robot en las asignaturas de la titulación, ya que todos estos factores presentan una p-valor menor que 0.0001. Otros factores como la importancia que se le da a la robótica en el currículo de un ingeniero, la aplicación a diferentes niveles, los inconvenientes docentes o la coordinación e integración en asignaturas presentan también una diferencia estadísticamente significativa entre los grupos de estudio y control $(\mathrm{p}<0.05)$. Tan solo en cuestiones más genéricas, como la aplicación de un aprendizaje basado en proyectos o el uso de instrucciones pautadas para construir el robot no se aprecian diferencias entre grupos.

En lo que se refiere a las asignaturas de aplicación y a las ventajas y desventajas que los alumnos del grupo de estudio indican en las preguntas abiertas cabe destacar los siguientes aspectos:

- Asignaturas: Se observa que los estudiantes identifican fácilmente múltiples asignaturas en las que se podría aplicar la propuesta como Control de robots, Automatismos y Control, Modelado y Simulación de Sistemas, Regulación Automática, Ingeniería de Control Automático, Microprocesadores, Electrónica de Potencia, Teoría de Máquinas y Mecanismos, o Resistencia de Materiales.

- Ventajas: Se relacionan mucho con la aplicabilidad de la propuesta. Algunos ejemplos son:

- "La construcción del robot lleva a la práctica muchos conocimientos de la carrera".

○ "En todos los puntos de mi titulación creo que es algo importante la robótica".

- "Orientación hacia una experiencia laboral más definida".

○ "Innovación y posibilidad de destacar la titulación sobre otras similares".

○ "Desarrollo de la identidad como ingeniero".

- "Se trata de un ámbito que concierne especialmente a los ingenieros electrónicos, existiendo numerosos másteres enfocados en la materia".

- "Ampliación de conocimientos y paso previo a posteriores mejoras por cuenta propia".

- Desventajas: Destaca el hecho de que 20 alumnos de 33 (más de un $60 \%$ ) no reportaron ninguna desventaja destacable, estando la mayoría de las desventajas centradas en las disponibilidades de recursos materiales y temporales, como, por ejemplo:

- "Acortamiento del temario en distintas asignaturas y recursos necesarios".

○ "Formación más específica, pudiendo dejar en menor medida otras actividades.".

- "Un coste elevado de implementación en las aulas". "El coste del material necesario para la fabricación de un robot desde cero, así como el software."

- “Administración del tiempo, aumentaría la dificultad de poder dar todos los temas del proyecto docente de cada asignatura, además de poder suponer una mayor dificultad para superar algunas asignaturas."

- Finalmente, en la última pregunta que permitía a los alumnos expresar cualquier cuestión adicional, se comentó lo siguiente: "La implementación de proyectos, en los que las asignaturas se enfocan en el desarrollo del mismo a lo largo de la formación universitaria del alumno, es una práctica habitual en el resto de Europa que ha dado muy buenos resultados, tanto a nivel académico cómo a nivel personal. Es un avance muy importante que cómo ya he comentado, fomenta mucho el interés individual y sirve como experiencia para decidir si la carrera que has elegido es la correcta, observando el uso práctico de la materia impartida.". 

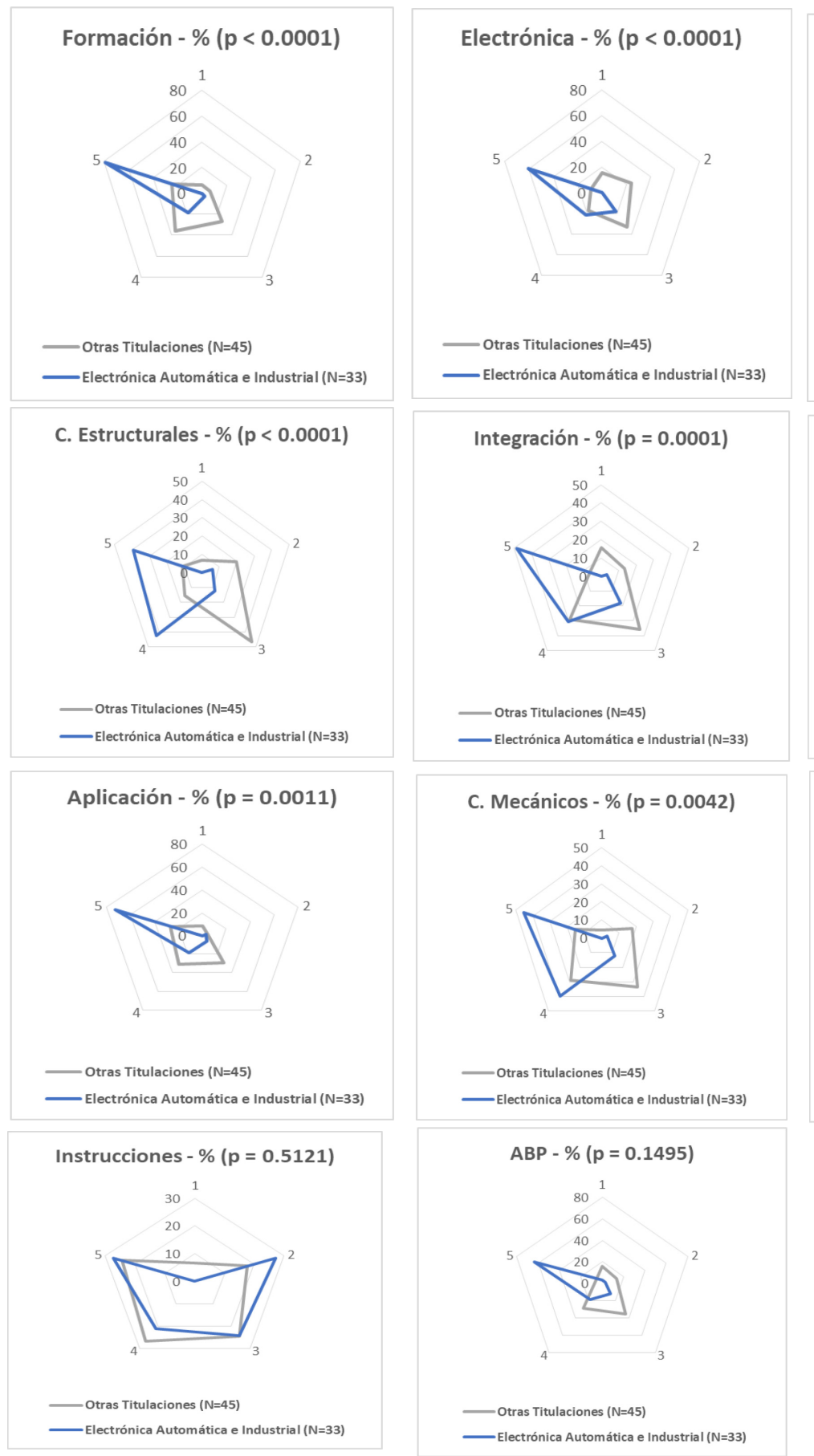

\section{Programación - \% $(p<0.0001)$}

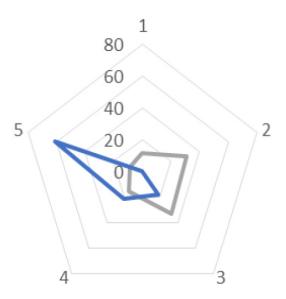

Otras Titulaciones ( $N=45)$

—Electrónica Automática e Industrial ( $\mathrm{N}=33$ )

Coordinación - \% $(p=0.0005)$

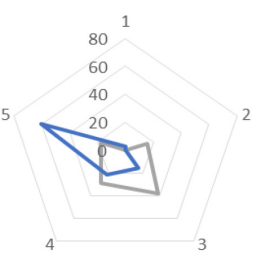

- Otras Titulaciones ( $N=45$ )

-Electrónica Automática e Industrial ( $N=33$ )

C. Mecánicos - \% ( $p=0.0042)$

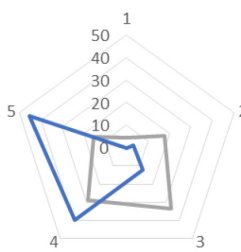

Importancia - \% ( $=0.0237)$

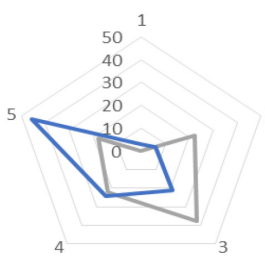

Otras Titulaciones ( $\mathrm{N}=45)$

—Electrónica Automática e Industrial ( $\mathrm{N}=33$ )

Otras Titulaciones $(\mathrm{N}=45)$

- Electrónica Automática e Industrial ( $N=33$ )

ABP - \% $(p=0.1495)$

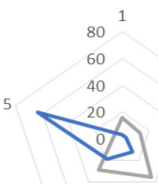

Inconvenientes $-\%(p=0.0386)$

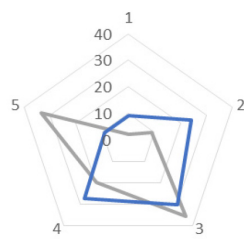

- Otras Titulaciones $(\mathrm{N}=45$

Electrónica Automática e Industrial ( $\mathrm{N}=33$ )

- Otras Titulaciones ( $\mathrm{N}=45$ )

- Electrónica Automática e Industrial ( $N=33$ )

Fig. 3 Resultados porcentuales de 12 preguntas en escala Likert ordenados de mayor a menor según las diferencias estadisticamente significativamente significativas entre grupos (p-valor del test de Chi-Cuadrado) 


\section{Conclusiones}

Como se ha desarrollado a lo largo de este trabajo, el uso de la robótica como material de apoyo sin importar el nivel educativo al que lo orientemos, trae consigo grandes ventajas, por lo que no es descabellado pensar que en un futuro próximo robótica y estudiantes compartirán aula en la mayoría de los centros de enseñanza.

A medida que avanza la tecnología y la sociedad, es necesario que lo hagan también los métodos de enseñanza, es por ello que el proyecto de innovación educativa "ROBOT-EDULPGC, Diseño, implementación y puesta en práctica de una plataforma modular de robótica educativa de bajo coste" en conjunto con algunos trabajos fin de grado de la Universidad de Las Palmas de Gran Canaria persigue esta reorientación académica, que no solo plantea la integración de la robótica, sino un aprendizaje basado en proyectos. Todo esto se plantea mediante una plataforma modular adaptable a la totalidad de niveles educativos, de fácil manejo y manteniendo un bajo coste para eliminar las barreras económicas y poder integrar la robótica en todos y cada uno de los centros de enseñanza.

En este artículo se ha presentado una propuesta de plataforma de robótica modular con hardware y software libre orientada a estudiante del Grado de Ingeniería en Electrónica y Automática (grupo de estudio) de la Universidad de Las Palmas de Gran Canaria. Según los resultados de la encuesta realizada se infieren las siguientes conclusiones principales de la visión de los estudiantes:

- Les gustaría recibir más formación en robótica.

- Consideran que la robótica es imprescindible en el currículo de un ingeniero.

- Valoran que la robótica es buena para fomentar la coordinación e integración entre asignaturas.

- Prefieren un aprendizaje basado en proyectos.

- Ven relativamente sencillo integrar la construcción de un robot modular sin distanciarse mucho del temario (integración de la electrónica, la programación y los elementos mecánicos).

Los resultados demuestran además que los estudiantes del grupo de estudio ven con mejores ojos la integración de la robótica y un aprendizaje basada en proyectos, que los estudiantes de otras titulaciones. Esto puede deberse en gran medida a que un ingeniero electrónico a lo largo del grado recibe formación en manejo de microprocesadores y en diferentes lenguajes de programación, que es sin duda la parte más compleja y esencial de la robótica.

\section{Agradecimientos}

Este trabajo se enmarca en el proyecto de innovación educativa PIE-2020-56 ROBOT-EDULPGC "Diseño, implementación y puesta en práctica de una plataforma modular de robótica educativa de bajo coste", proyecto concedido en la Convocatoria de Proyectos de Innovación Educativa 2020 de la Universidad de Las Palmas de Gran Canaria.

\section{Referencias}

AISOY ESPAÑA. (2009). Aisoy, robot Mentor Personal. <https://aisoy.es/>. [Consulta: 16 de marzo de 2020]

ALCAIDE, MaA y DE LA POZA, (2019). "El uso de los dispositivos electrónicos móviles como herramienta docente de una asignatura de Grado. IN-RED 2019 V Congreso de Innovación Educativa y Docencia en Red. Valencia: Universitat politècnica de valencia [Consulta: 5 de marzo de 2020] 
ANGULO, C. (2017). Usos y beneficios de la robótica en las aulas <https://www.upc.edu/latevaupc/usos-ybeneficios-robotica-las-aulas/> [Consulta: 19 de marzo de 2020]

ARDUINO. (2006). Arduino. <https://www.arduino.cc/>. [Consulta: 16 de marzo de 2020]

CLEMENTONI. (2017). DOC, el Robot Educativo con voz. < $<$ https://www.clementoni.com/es/55176-doc-el-roboteducativo-con-voz/> [Consulta: 15 de marzo de 2020]

BQ. (2015). Zowi, el robot inteligente y educativo para niños. <https://www.bq.com/es/zowi.> [Consulta: 16 de marzo de 2020]

DF ROBOTS. (2018). Micro: Maqueen micro: bit Plataforma de robot de programación educativa< https://www.dfrobot.com/product-1783.html $>$ [Consulta: 8 de febrero de 2020]

EDELVIVES. (2019). Robot Next 2.0. <https://www.edelvives.com/es/Catalogo/p/robot-primaria-next> [Consulta: 2 de febrero de 2020]

ESCORNABOT. (2015). Escornabot. <http://escornabot.com/web/> [Consulta: 12 de febrero de 2020]

JUGUETRÓNICA. (2016). Productos $<$ https://www.juguetronica.com/products/1413707/coder-mip $>$ [Consulta: 12 de febrero de 2020]

MAKEBLOCK. (2012). Venta de productos Makeblock y Compatibles en España. <https://makeblock.es/> [Consulta: 8 de febrero de 2020]

MICROBIT. (2000). BBC micro:bit. $<$ https://microbit.org/>[Consulta: 8 de febrero de 2020]

MICROBRIC. (2014). Meet Edison. <https://meetedison.com/> [Consulta: 8 de febrero de 2020]

MOBSYA. (2011). Thymio, the educational robot to learn, code and create. $<$ https://www.thymio.org/> [Consulta: 6 de febrero de 2020]

PERALTA BUITRAGO G. (2015). Una estrategia en el desarrollo de la creatividad y las capacidades en educación en tecnología $<$ http://docplayer.es/57947451-Robotica-educativa-una-estrategia-en-el-desarrollo-de-la-creatividad-ylas-capacidades-en-educacion-en-tecnologia.html $>$ [Consulta: 16 de marzo de 2020]

PINTO SALAMANCA, ML et al. (2009). "Uso de la robótica educativa como herramienta en los procesos de enseñanza" Grupo de Investigación en Robótica y Automatización Industrial, GIRA. Colombia: Universidad Pedagógica y Tecnológica de Colombia, [Consulta: 6 de febrero de 2020]

QUEVEDO, E. et al. (2018). Desarrollo de Competencias STEAM mediante Robótica Marina Educativa de Bajo Coste. II Congreso Internacional en Tecnologías e Innovación Educativa. Valencia: Universidad Internacional de Valencia.

RO-BOTICA. (2015). Robots infantiles programables. <http://ro-botica.com/tienda/BEE-BOT/Robots-infantilesprogramables-TTS/. [Consulta: 6 de febrero de 2020]

ROBOT PARA NIÑOS. (2017). Ratón robot programable Code and Go robot $<\underline{\mathrm{https}}$ ://www.robotsparaninos.com/code-and-go-robot-mouse-activity-set/>[Consulta: 13 de febrero de 2020]

THE LEGO GROUP. (2018). Caja de herramientas creativas 17101. <https://www.lego.com/es-es/product/boostcreative-toolbox-17101>. [Consulta: 6 de marzo de 2020]

THE LEGO GROUP. (2014) LEGO Mindstorms EV3 3131. <https://www.lego.com/es-es/product/lego-mindstormsev3-31313> [Consulta: 18 de febrero de 2020]

VEGA-MORENO, D, et al. (2015). "Project-based learning using robots with open source hardware and software" Jornadas Iberoamericanas de Innovación Educativa en el ámbito de las TIC. Gran Canaria: Universidad de las Palmas de Gran Canaria [Consulta: 25 de febrero de 2020]

WOWWEE. (2016). Coji. <https://wowwee.com/coji> [Consulta: 17 de marzo de 2020] 